\title{
Dynamics of serotype 14 Streptococcus pneumoniae population causing acute respiratory infections among children in China (1997-2012)
}

\author{
Mingming $\mathrm{He}^{\dagger}$, Kaihu Yao ${ }^{\dagger}$, Wei Shi ${ }^{\dagger}$, Wei Gao, Lin Yuan, Sangjie Yu and Yonghong Yang ${ }^{*}$
}

\begin{abstract}
Background: In the last decade, the Streptococcus pneumoniae population has changed, mainly due to the abuse of antibiotics. The aim of this study was to determine the genetic structure of 144 S. pneumonia serotype 14 isolates collected from children with acute respiratory infections during 1997-2012 in China.

Methods: All isolated pneumococci were tested for their sensitivity to 11 kinds of antibiotics with the E-test method or disc diffusion. The macrolides resistance genes ermB and mefA, as well as the

sulfamethoxazole-trimethoprim resistance gene dihydrofolate reductase (DHFR) were detected by polymerase chain reaction (PCR). The sequence types (STs) were analyzed with multilocus sequence typing (MLST).

Results: From 1997 to 2012, the percentage of serotype 14 S. pneumonia isolates in the whole isolates increased. All of the 144 serotype 14 S. pneumonia isolates were susceptible to amoxicillin-clavulanic acid, vancomycin and levofloxacin. No penicillin resistant isolate was found, and the intermediate rate was as low as $0.7 \%$. Erythromycin resistance was confirmed among 143 isolates. The ermB gene was determined in all erythromycin resistant isolates, and the mefA gene was positive additionally in 13 of them. The non-susceptibility rate to the tested cephalosporins increased from 1997-2012. All trimethoprim-resistant isolates contained the lle100-Leu mutation. Overall, 30 STs were identified, among which ST876 was the most prevalent, followed by ST875. During the study period, the percentage of CC876 increased from $0 \%$ in 1997-2000 to $96.4 \%$ in 2010-2012, whereas CC875 decreased from 84.2 to $0 \%$. CC876 showed higher non-susceptibility rates to $\beta$-lactam antibiotics than CC875.

Conclusion: The percentage of serotype 14 S. pneumonia isolates increased over time in China. The increase of resistance to $\beta$-lactam antibiotics in this serotype isolates was associated with the spread of CC876.
\end{abstract}

Keywords: Streptococcus pneumoniae, Serotypes, Antibiotic resistance, Children, Epidemiology

\section{Background}

Streptococcus pneumoniae (S. pneumonia) is a leading cause of bacterial infections in infants and young children. World Health Organization (WHO) estimated that 1.6 million people die of pneumococcal diseases annually, including $0.7-1$ million children aged $<5$ years mostly from developing countries [1]. Management of $S$. pneumonia infections has been complicated by the emergence of multiple antimicrobial drug-resistant strains [2].

\footnotetext{
*Correspondence: shiwei613115@126.com

${ }^{\dagger}$ Equal contributors

Key Laboratory of Major Diseases in Children and National Key Discipline of Pediatrics, Ministry of Education, Beijing Pediatric Research Institute, Beijing Children's Hospital Affiliated to Capital Medical University, Beijing, P. R. China
}

More than 92 serotypes have been identified based on the discrepancy of capsular polysaccharide antigens. Before the introduction of the pneumococcal conjugate vaccines, serotype $14 \mathrm{~S}$. pneumonia was one of the most common types worldwide $[3,4]$ and it was usually one common type of antibiotic resistant isolates [5]. Penicillinresistant and multidrug-resistant pneumococci were known to be restricted to serogroups 23, 6, 19 and serotype 14 , which were particularly associated with the infectious diseases in children [6-8]. Spain ${ }^{14-5}(\mathrm{ST} 18)$, England $^{14-9}(\mathrm{ST} 9), \mathrm{CSR}^{14-10}(\mathrm{ST} 20)$, Tennessee ${ }^{14-18}$ (ST67), Denmark $^{14-32}$ (ST230) and Netherlands ${ }^{14-35}$ (ST124) were internationally disseminated antimicrobial resistant clones described by the Pneumococcal Molecular Epidemiology 
Network (PMEN) (http://www.sph.emory.edu/PMEN/ index.html).

We reported previously some international spread drug resistant clones in serotype $19 \mathrm{~F}$ and $23 \mathrm{~F}$ which had been identified in China with increasing frequency $[9,10]$. The current study is conducted to analyze the antimicrobial resistance and population biology of serotype 14S. pneumonia isolates in China.

\section{Methods}

\section{Pneumococcal isolates}

From 1997 to 2012, a total of 1984 S. pneumonia isolates were collected from children $<5$ years old with the diagnosis of acute upper respiratory infection in Beijing, Shanghai, Guangzhou, Shenzhen, Chongqing, Xi'an of China. The patients suffering from "lower respiratory tract infections" like pneumonia were excluded because we can not ensure the isolates were from lower respiratory tract or from the nasopharynx. The details of these S. pneumoniae collections have been published previously [11-16]. A total of 144 isolates were identified as serotype 14 based on quellung reaction, which was performed using the Pneumotest Kit (Statens Serum Institut, Copenhagen, Denmark). The isolates were cultured from nasopharyngeal specimens, except those collected from Beijing in 2006-2008 and from Shenzhen in 20092011, which were cultured from hypopharyngeal aspirate specimens.

All isolates were taken as part of standard patient care. A parent and/or legal guardian of each participant signed a written informed consent document before enrollment and before any study procedure was performed. This study was viewed and approved by the Ethics Committee of Beijing Children's Hospital Affiliated to Capital Medical University. No ethical problems existed in this study.

\section{Antimicrobial susceptibility}

The minimum inhibitory concentrations (MICs) of all isolates were determined for penicillin, amoxicillin-clavulanic acid, ceftriaxone, cefuroxime, erythromycin, imipenem, levofloxacin, and vancomycin using E-test strips (AB Biodisk, Solna, Sweden) [17], and their susceptibility to tetracycline, sulfamethoxazole-trimethoprim, and chloramphenicol was assessed with disc diffusion tests (Oxoid). The breakpoints were adopted in accordance with the Clinical and Laboratory Standards Institute 2012 criteria [18]. Streptococcus pneumoniae ATCC49619 was used as the reference strain in the susceptibility tests. Isolates were considered multidrug resistant if they were not susceptible to three or more classes of antimicrobials.

\section{Macrolide-resistance genes}

Chromosomal DNA was extracted from overnight cultures of S. pneumonia isolates grown on $5 \%$ trypticase soy agar (Oxoid Ltd, Basingstoke, England) using the SiMax ${ }^{\text {Tu }}$ Genomic DNA Extraction Kit (SBS Genetech Co., Ltd), according to the manufacturer's instructions. The macrolide-resistance genes ermB and $m e f A$ were detected by polymerase chain reaction (PCR) for all of the erythromycin non-susceptible strains, the primers and PCR procedures were previously described [19].

\section{Multilocus sequence typing (MLST)}

All strains were characterized with multilocus sequence typing (MLST). Bacterial chromosomal DNA was extracted as described above. The PCR fragments of the seven MLST genes (aroE, gdh, gki, recP, $s p i$, xpt, and $d d l$ ) were amplified from the chromosomal DNA. The products were sent to BGI Company (Beijing, China) for sequencing on both strands. The STs were determined by comparing the allelic profiles with the recognized STs at the MLST website (http://spneumoniae.mlst.net). The new alleles and allelic profiles identified in the present study have been submitted to the MLST database for name assignment. The eBURST v3 software (available at http://www.mlst.net) was used to estimate the relationships among the isolates and to assign

Table 1 Susceptibility and MICs of 144 pneumococcal isolates to 11 antibiotics

\begin{tabular}{|c|c|c|c|c|c|}
\hline \multirow[t]{2}{*}{ Antibiotics } & \multicolumn{2}{|c|}{ Susceptibility } & \multicolumn{3}{|c|}{$\mathrm{MIC}(\mu \mathrm{g} / \mathrm{ml})$} \\
\hline & $\mid(\%)$ & $R(\%)$ & $50 \%$ & $90 \%$ & Range \\
\hline PEN & 0.7 & 0 & 0.5 & 1 & $0.004-3$ \\
\hline $\mathrm{AMC}$ & 0 & 0 & 0.38 & 1 & $0.008-2$ \\
\hline CXM & 43.8 & 7.6 & 1.5 & 3 & $0.008-6$ \\
\hline CRO & 6.9 & 1.4 & 0.38 & 1 & $0.004-8$ \\
\hline IPM & 49.3 & 0 & 0.094 & 0.19 & $0.004-0.38$ \\
\hline ERY & 0 & 99.3 & $>256$ & $>256$ & $0.125->256$ \\
\hline LVX & 0 & 0 & 0.5 & 0.75 & $0.25-1.5$ \\
\hline VAN & 0 & 0 & 0.5 & 0.75 & $0.094-1$ \\
\hline TCY & 28.5 & 56.9 & - & - & - \\
\hline SXT & 0 & 47.2 & - & - & - \\
\hline $\mathrm{CHL}$ & 0 & 15.3 & - & - & - \\
\hline
\end{tabular}

PEN penicillin, $A M C$ amoxicillin-clavulanic acid, CXM cefuroxime, $C R O$ ceftriaxone, IPM imipenem, ERY erythromycin, $L V X$ levofloxacin, VAN vancomycin, $T C Y$ tetracycline, $C H L$ chloramphenicol,

SXT trimethoprim-sulfamethoxazole, - no data for disk diffusion test, I intermediate, $R$ resistant, MIC50 minimum inhibitory concentration at which $50 \%$ of the strains were inhibited, MIC90 minimum inhibitory concentration at which $90 \%$ of the strains were inhibited, MIC range range of minimum inhibitory concentration 
strains to a clonal complex (CC) using the stringent group definition of six of seven shared alleles.

\section{Dihydrofolate reductase genes}

The dihydrofolate reductase (DHFR) genes were amplified for all isolates with primers previously reported [20] and then sequenced. The sequences were analyzed and compared to each other by MEGA4.1 software.

\section{Statistical analysis}

The antibiotic susceptibility data were analyzed using WHONET 5.6 software as recommended by the WHO. The $X^{2}$ test, performed with the SPSS software v. 13.0 (SPSS Inc. USA), was used for statistical comparisons. For effective comparison, the study period was divided into five stages: 1997-2000, 2001-2003, 2004-2006,
2007-2009 and 2010-2012. A two-tailed cut-off of $P<0.05$ indicated statistical significance.

\section{Results}

Frequency of serotype $14 \mathrm{~S}$. pneumonia isolates over time

During the study period, serotype 14 isolates was identified in $7.3 \%(144 / 1984)$ of all of the S. pneumonia strains. The frequencies in different stages were $3.2 \%$ $(19 / 593)$ in $1997-2000,5.5 \%(38 / 691)$ in $2001-2003$, $7.1 \%(24 / 338)$ in 2004-2006, $22 \%(35 / 159)$ in 20072009, $13.8 \%(28 / 203)$ in $2010-2012 \quad\left(X^{2}=82.069\right.$, $P<0.05)$.

\section{Antibiotic susceptibility}

The susceptibility and the MICs of the isolates against 11 antibiotics were presented in Table 1 . All of the 144

Table 2 Susceptibility of the 144 serotype 14 isolates to eight antimicrobials throughout the study period

\begin{tabular}{|c|c|c|c|c|c|c|c|}
\hline Antibiotics & Susceptibility and MIC & $\begin{array}{l}1997-2000 \\
(n=19)\end{array}$ & $\begin{array}{l}2001-2003 \\
(n=38)\end{array}$ & $\begin{array}{l}2004-2006 \\
(n=24)\end{array}$ & $\begin{array}{l}2007-2009 \\
(n=35)\end{array}$ & $\begin{array}{l}2010-2012 \\
(n=28)\end{array}$ & $\begin{array}{l}\text { Total } \\
(n=144)\end{array}$ \\
\hline \multirow[t]{4}{*}{ PEN } & $1 \%$ & 0 & 0 & 0 & 2.9 & 0 & 0.7 \\
\hline & $\operatorname{MIC50}(\mu \mathrm{g} / \mathrm{ml})$ & 0.016 & 0.032 & 0.5 & 0.75 & 0.5 & 0.5 \\
\hline & $\operatorname{MIC} 90(\mu \mathrm{g} / \mathrm{ml})$ & 0.75 & 0.5 & 1 & 1.5 & 1 & 1 \\
\hline & MIC Range( $\mu \mathrm{g} / \mathrm{ml})$ & $0.004-1.5$ & $0.008-0.75$ & $0.012-1.5$ & $0.008-3$ & $0.25-1.5$ & $0.004-3$ \\
\hline \multirow[t]{3}{*}{ AMC } & $\operatorname{MIC50}(\mu \mathrm{g} / \mathrm{ml})$ & 0.016 & 0.023 & 0.5 & 0.5 & 0.5 & 0.38 \\
\hline & $\operatorname{MIC90}(\mu \mathrm{g} / \mathrm{ml})$ & 0.75 & 0.5 & 1 & 1 & 0.75 & 1 \\
\hline & MIC Range( $\mu \mathrm{g} / \mathrm{ml})$ & $0.008-1$ & $0.008-1$ & $0.016-2$ & $0.008-1$ & $0.25-1.5$ & $0.008-2$ \\
\hline \multirow[t]{5}{*}{ CXM } & $1 \%$ & 10.5 & 13.2 & 50.0 & 65.7 & 75.0 & 43.8 \\
\hline & $\mathrm{R} \%$ & 0 & 0 & 4.2 & 20.0 & 10.7 & 7.6 \\
\hline & $\operatorname{MIC50}(\mu \mathrm{g} / \mathrm{ml})$ & 0.023 & 0.032 & 1.5 & 2 & 2 & 1.5 \\
\hline & $\operatorname{MIC90}(\mu \mathrm{g} / \mathrm{ml})$ & 2 & 1.5 & 3 & 4 & 4 & 3 \\
\hline & MIC Range( $\mu \mathrm{g} / \mathrm{ml})$ & $0.016-2$ & $0.008-2$ & $0.016-4$ & $0.032-4$ & $0.25-6$ & $0.008-6$ \\
\hline \multirow[t]{5}{*}{$\mathrm{CRO}$} & $1 \%$ & 0 & 0 & 4.2 & 17.1 & 10.7 & 6.9 \\
\hline & $\mathrm{R} \%$ & 0 & 0 & 0 & 0 & 7.1 & 1.4 \\
\hline & $\operatorname{MIC50}(\mu \mathrm{g} / \mathrm{ml})$ & 0.016 & 0.032 & 0.5 & 0.75 & 0.5 & 0.38 \\
\hline & MIC90( $\mu \mathrm{g} / \mathrm{ml})$ & 0.38 & 0.38 & 1 & 1.5 & 2 & 1 \\
\hline & MIC Range( $\mu \mathrm{g} / \mathrm{ml})$ & $0.004-0.5$ & $0.008-0.75$ & $0.016-1.5$ & $0.008-2$ & $0.25-8$ & $0.004-8$ \\
\hline \multirow[t]{4}{*}{ IPM } & $1 \%$ & 10.5 & 18.4 & 50.0 & 62.9 & 100 & 49.3 \\
\hline & $\operatorname{MIC50}(\mu \mathrm{g} / \mathrm{ml})$ & 0.012 & 0.032 & 0.094 & 0.125 & 0.19 & 0.094 \\
\hline & $\operatorname{MIC90}(\mu \mathrm{g} / \mathrm{ml})$ & 0.125 & 0.125 & 0.19 & 0.19 & 0.25 & 0.19 \\
\hline & MIC Range( $\mu \mathrm{g} / \mathrm{ml})$ & $0.004-0.125$ & $0.004-0.19$ & $0.012-0.19$ & $0.012-0.19$ & $0.125-0.38$ & $0.004-0.38$ \\
\hline \multirow[t]{2}{*}{$\mathrm{TCY}$} & $1 \%$ & 0 & 5.3 & 8.3 & 40.0 & 82.1 & 28.5 \\
\hline & $\mathrm{R} \%$ & 94.7 & 86.8 & 75.0 & 28.6 & 10.7 & 56.9 \\
\hline \multirow[t]{2}{*}{ SXT } & $1 \%$ & 0 & 0 & 0 & 0 & 0 & 0 \\
\hline & $\mathrm{R} \%$ & 84.2 & 86.8 & 45.8 & 20.0 & 3.6 & 47.2 \\
\hline $\mathrm{CHL}$ & $\mathrm{R} \%$ & 52.6 & 18.4 & 20.8 & 0 & 0 & 15.3 \\
\hline
\end{tabular}

PEN penicillin, AMC amoxicillin-clavulanic acid, CXM cefuroxime, CRO ceftriaxone, IPM imipenem, TCY tetracycline, CHL chloramphenicol, SXT trimethoprimsulfamethoxazole, - no data for disk diffusion test, $I$ intermediate, $R$ resistant, MIC50 minimum inhibitory concentration at which $50 \%$ of the strains were inhibited, MIC90 minimum inhibitory concentration at which $90 \%$ of the strains were inhibited, MIC range range of minimum inhibitory concentration 
Table 3 The amino acid mutation of dihydrofolate reductase of trimethoprim-sulfamethoxazole-susceptible and -resistant isolates

\begin{tabular}{lll}
\hline $\begin{array}{l}\text { Reference } \\
\text { sequence }^{a}\end{array}$ & $\begin{array}{l}\text { Sequence types } \\
\text { of SXT-susceptible } \\
\text { isolates }\end{array}$ & Sequence types of SXT-resistant isolates \\
\hline
\end{tabular}

\begin{tabular}{|c|c|c|c|c|c|c|c|c|c|c|c|c|c|c|c|c|c|c|c|c|c|}
\hline sites & $\begin{array}{l}\text { Amino } \\
\text { acid }\end{array}$ & $\begin{array}{l}\text { S1 } \\
\text { (6) }\end{array}$ & $\begin{array}{l}\text { S2 } \\
\text { (15) }\end{array}$ & $\begin{array}{l}\text { S3 } \\
\text { (55) }\end{array}$ & $\begin{array}{l}\text { R1 } \\
\text { (1) }\end{array}$ & $\begin{array}{l}\text { R2 } \\
\text { (1) }\end{array}$ & $\begin{array}{l}\text { R3 } \\
\text { (1) }\end{array}$ & $\begin{array}{l}\text { R4 } \\
\text { (1) }\end{array}$ & $\begin{array}{l}\text { R5 } \\
\text { (1) }\end{array}$ & $\begin{array}{l}\text { R6 } \\
\text { (1) }\end{array}$ & $\begin{array}{l}\text { R7 } \\
\text { (1) }\end{array}$ & $\begin{array}{l}\text { R8 } \\
(1)\end{array}$ & $\begin{array}{l}\text { R9 } \\
\text { (2) }\end{array}$ & $\begin{array}{l}\text { R10 } \\
\text { (2) }\end{array}$ & $\begin{array}{l}\text { R11 } \\
\text { (3) }\end{array}$ & $\begin{array}{l}\text { R12 } \\
\text { (4) }\end{array}$ & $\begin{array}{l}\text { R13 } \\
\text { (5) }\end{array}$ & $\begin{array}{l}\text { R14 } \\
\text { (6) }\end{array}$ & $\begin{array}{l}\text { R15 } \\
\text { (11) }\end{array}$ & $\begin{array}{l}\text { R16 } \\
\text { (12) }\end{array}$ & $\begin{array}{l}\text { R17 } \\
\text { (15) }\end{array}$ \\
\hline
\end{tabular}

1 Met

\section{$13 \mathrm{Glu}$}

14 Glu

20 Glu

26 His

$32 \mathrm{Gln}$

53 Met

60 Lys

70 Pro

73 Lys

74 Ile

75 Asp

78 Ala

79 Thr

81 Gln

82 Asp

$83 \mathrm{Val}$

$84 \mathrm{Gln}$

85 Ser

88 Asp

$91 \mathrm{Gln}$

92 Asp

$94 \mathrm{Glu}$

99 lle

100 lle 105 Ile

108 Ala

110 Glu

111 Pro

$116 \mathrm{Val}$

120 His

124 Arg

130 Tyr

133 Glu

134 Glu

135 Leu

137 Leu

138 Ser

139 Leu

$142 \mathrm{Thr}$

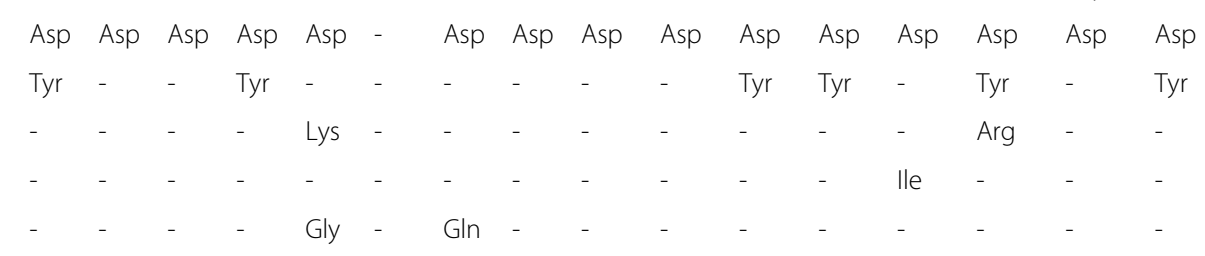


Table 3 The amino acid mutation of dihydrofolate reductase of trimethoprim-sulfamethoxazole-susceptible and -resistant isolates (Continued)

\begin{tabular}{|c|c|c|c|c|c|c|c|c|c|c|c|c|c|c|c|c|c|c|c|c|c|}
\hline 144 & Ser & - & - & - & - & - & - & - & - & Ala & - & - & - & - & - & - & - & - & - & - & - \\
\hline 147 & Phe & - & - & - & - & - & - & Ser & Ser & Ser & Ser & Ser & Ser & Ser & - & Ser & Ser & Ser & - & Ser & Ser \\
\hline 148 & Tyr & - & - & - & - & - & - & - & - & - & - & - & - & - & - & Phe & - & - & - & - & - \\
\hline 149 & Ala & - & - & - & - & - & - & Thr & Thr & Thr & Thr & Thr & Thr & Thr & Val & Thr & Thr & Thr & - & Thr & Thr \\
\hline 150 & Lys & - & - & - & - & - & - & - & - & Arg & - & - & - & - & - & - & - & - & - & - & - \\
\hline 155 & Pro & - & - & - & - & - & - & - & - & Ala & - & - & - & - & - & - & - & - & - & - & - \\
\hline 160 & Ile & - & - & - & - & - & - & - & - & - & Thr & - & - & - & - & - & - & - & - & - & - \\
\hline 161 & Gln & - & - & - & - & - & - & - & - & Glu & - & - & - & - & - & - & - & - & - & - & - \\
\hline 162 & Tyr & - & - & - & - & - & Cys & - & - & - & - & - & - & - & - & - & - & - & - & - & - \\
\hline
\end{tabular}

S SXT-susceptible isolates, $R$ SXT-resistant isolates, - The same with the ATCC49619

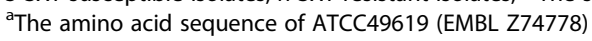

strains were susceptible to amoxicillin-clavulanic acid, vancomycin and levofloxacin. Only one isolate was intermediate to penicillion (MIC: $3 \mu \mathrm{g} / \mathrm{mL}$ ). The nonsusceptible rates to cefuroxime, ceftriaxone, imipenem and tetracycline were $51.4 \%, 8.3 \%, 49.3 \%$ and $85.4 \%$, respectively. A total of 143 (99.3\%) isolates were resistant to erythromycin with high MICs (139 isolates had a $\mathrm{MIC} \geq 256 \mu \mathrm{g} / \mathrm{mL}$ ). The resistance rates to trimethoprimsulfamethoxazole and chloramphenicol were $47.2 \%$ and $15.3 \%$, respectively. About $52.1 \%(75 / 144)$ of all pneumococcal isolates were MDRSP, most of which were co-resistance to erythromycin, tetracycline, and sulfamethoxazole-trimethoprim.

The resistance rate of serotype $14 \mathrm{~S}$. pneumonia isolates against $\beta$-lactam antibiotics showed an increasing trend (Table 2). From 1997 to 2012, the nonsusceptibility rates to ceftriaxone, cefuroxime and imipenem increased. By contrast, the non-susceptibility rate to sulfamethoxazole-trimethoprim and chloramphenicol decreased from $84.2 \%$ in $1997-2000$ to $3.6 \%$ in $2010-$ 2012 and from 52.6 to $0 \%$ in 2007-2009 and 20102012, respectively.

\section{Macrolide resistance genes}

The $\operatorname{erm} B$ gene was detected in all of the 143 erythromycin resistant isolates, and the mefA gene was detected additionally in $13(9.1 \%)$ isolates.

\section{DHFR amino acid sequences}

The DHFR sequences of the 144 strains were compared with that of S. pneumonia ATCC 49619 (EMBL Z74778). The DHFR coding sequence, consisting of 504 nucleotides, encoding 168 amino acids, in trimethoprimsusceptible isolates showed a divergence of 3 to 7 nucleotides (0.6 to $1.4 \%$ ) compared with the ATCC 49619 isolate, resulting in 2 to 4 variations in amino acid sequence (1.2 to $2.4 \%)$. DHFR coding sequences of trimethoprim-resistant isolates showed a 37 - to 38nucleotide divergence (7.3 to $7.5 \%$ ) from S. pneumonia ATCC 496 19, resulting in 2 to 30 differences (1.2 to $17.9 \%$ ) in amino acid sequence (Table 3 ). In the present data, Ile100-Leu variation was determined in all of the trimethoprim-resistant isolates and none of the trimethoprim-susceptible isolate. Frequent variation in trimethoprim-resistant isolates with no change in trimethoprim-susceptible isolates included Glu20-Asp (97.1\% resistant isolates), Glu94-Asp (83.8 \% resistant isolates), Leu135-Phe (97.1\% resistant isolates).

\section{MLST}

MLST analysis revealed 30 STs, the most common of which were ST876 (49/144, $34.0 \%)$, ST875 (41/144, $28.5 \%), \operatorname{ST} 790$ (8/144, $5.6 \%), \operatorname{ST} 143$ (6/144, $4.2 \%)$ and ST777 (6/144, $4.2 \%)$. eBURST analysis revealed the presence of six CCs and six singletons (Fig. 1). CC876 was the most common group comprising 8 STs and accounted for $39.6 \%$ of all strains while CC875 accounted for $32.6 \%$ of the studied strains.

Figure 2 shows the distribution of the common CCs in serotype $14 \mathrm{~S}$. pneumonia strains during the study period. From 1997 to 2012, the frequency of CC876 increased from $0 \%$ in $1997-2000$ to $25 \%$ in 2004-2006, and reached $96.4 \%$ in 2010-2012, whereas CC875 decreased from 84.2 to $33.3 \%$ and finally to $0 \%$ in the corresponding periods.

Significant differences in $\beta$-lactam antibiotic resistance between the predominant CCs were found in this study (Table 4). All CC875 strains were susceptible to $\beta$-lactam antibiotics while CC876 and CC143 isolates showed high $\beta$-lactam antibiotic nonsusceptibility and MICs (Fig. 3). By contrast, CC875 were significantly more resistant to trimethoprimsulfamethoxazole and chloramphenicol than CC876. 


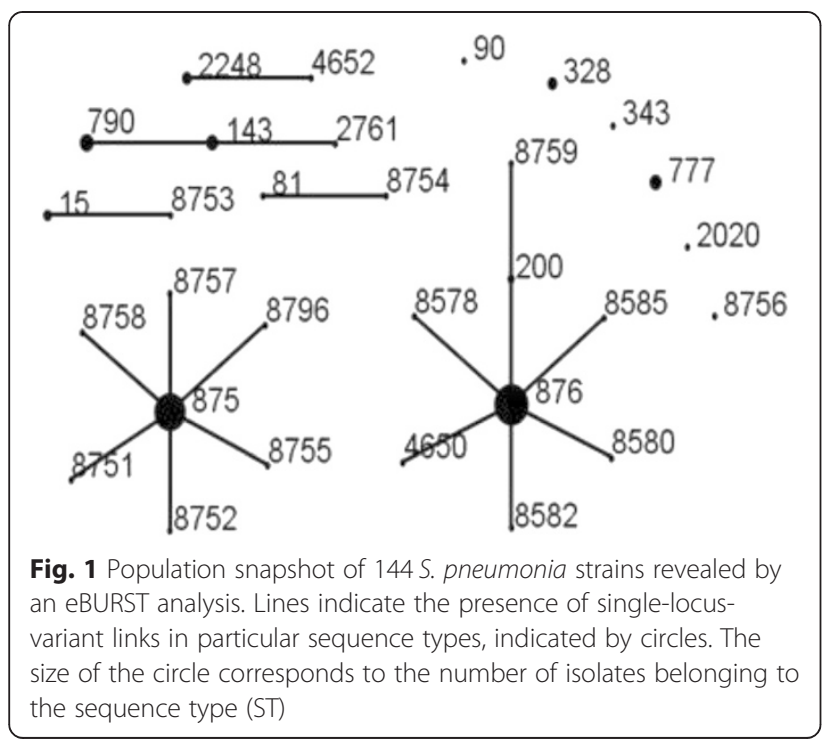

Both clones exhibited similar resistance to erythromycin and tetracycline.

\section{Discussion}

The present study showed that serotype 14 isolates was not very common in all of the S. pneumonia $(7.3 \%)$ strains isolated from Chinese children. But its percentage in the whole $S$. pneumonia isolates over time increased from $3.2 \%$ in 1997-2000 to $13.8 \%$ in 2010 2012. Epidemiology data in a previous report showed that the percentage of serotype 14 isolates was usually lower than $10.0 \%$ [21]. From January 2005 to December 2006, a total of 451 S. pneumonia isolates were collected from 9 study centers in 8 cities across China, of which the most prevalent serotypes in descending order were $19 \mathrm{~F}(42.1 \%), 19 \mathrm{~A}(11.8 \%), 14$ (7.5 \%), 6B (7.3\%), $23 \mathrm{~F}$ (5.8\%), and 15 (4.4\%) [22]. Our previous study also revealed that among 338 pneumococcal isolates, the most common were $19 \mathrm{~F}$ (55.6 \%), 19A (13.9 \%), $23 \mathrm{~F}$ (10.1\%), 6B (4.7\%), and 14 (3.6 \%) [23]. But among the isolates causing invasive pneumococcal disease (IPD) in children, serotype 14 may play a more significant role. In a study [14] conducted from 2006 to 2008 in 11 hospitals across China, $171 \mathrm{~S}$. pneumonia isolates collected from children suffering from IPD under the age of 14 were analyzed for serotype distribution, and the most prevalent serotype was 19 F (19.9 \%), followed by serotype $14(19.3 \%)$. In another previous study in China [12], a total of 61 invasive strains were isolated in Shenyang, the serotype distribution of those isolates were 19A (41.0 \%), 14 (19.7 \%), $19 \mathrm{~F}(11.5 \%)$ and $23 \mathrm{~F}$ $(9.8 \%)$. Data from developed countries before the introduction of the conjugated vaccines indicated that the proportion of serotype 14 was 15.7 to $19.8 \%$ [8, 24]. In September 2008, the 7-valent pneumococcal conjugated vaccine (PCV7) became available for the private sector in China. However, the vaccination rate is very low (less than $1.0 \%$ ). Thus, the frequency of serotype 14 did not decrease in 2012, and even increased. During the investigation period, the susceptibility to beta-lactam antibiotics of serotype 14 isolates decreased. We also found a significant clonal shift of serotype 14 strains from CC875 to CC876 over time. Given the discrepancy of antibiotic resistance between these two CCs, the genotype replacement was deduced to be caused by the antibiotic selective pressure. The spread of the highly resistant CC876 could be driven by the selective

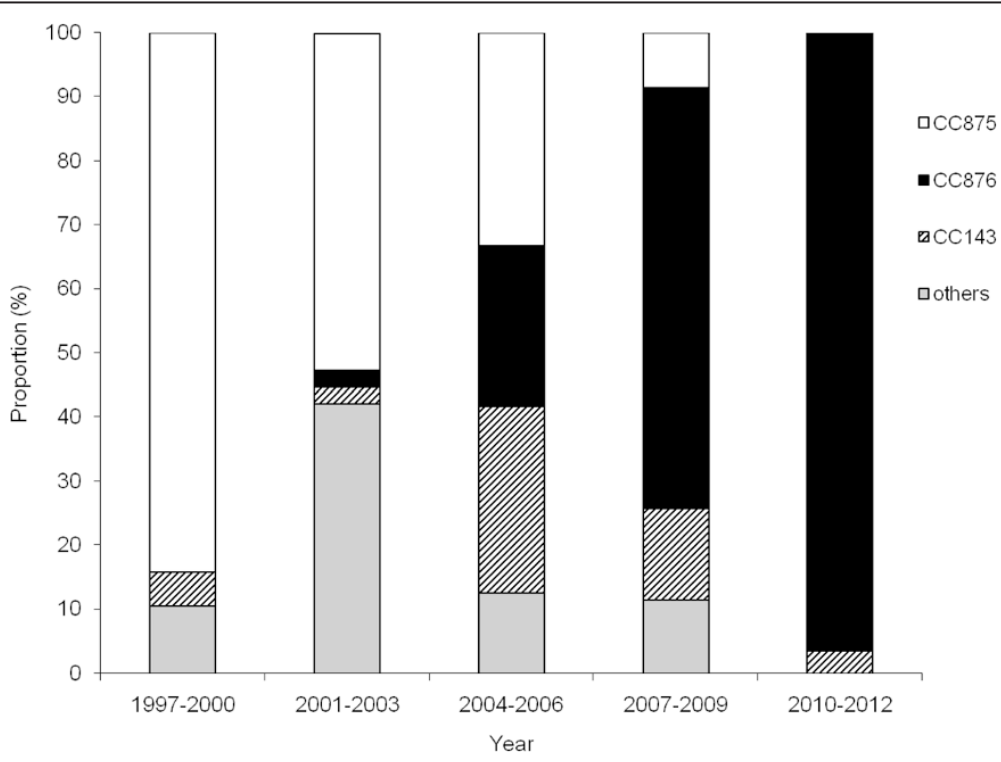

Fig. 2 Distribution of the common CCs of serotype 14S. pneumonia isolates during the study period 
Table 4 Difference between the antimicrobial susceptible profiles of CC876 and CC875 of serotype 14 S. pneumonia

\begin{tabular}{|c|c|c|c|c|c|c|}
\hline Antibiotics & Susceptibility and MIC & CC876 $(n=57)$ & CC875 $(n=47)$ & $\operatorname{CC143}(n=15)$ & Others $(n=25)$ & Total \\
\hline \multirow[t]{4}{*}{ PEN } & $1 \%$ & 0 & 0 & 6.7 & 0 & 0.7 \\
\hline & $\operatorname{MIC50}(\mu \mathrm{g} / \mathrm{mL})$ & 0.75 & 0.016 & 1 & 0.125 & 0.5 \\
\hline & MIC90( $\mu \mathrm{g} / \mathrm{mL})$ & 1 & 0.023 & 1.5 & 0.75 & 1 \\
\hline & MIC Range( $\mu \mathrm{g} / \mathrm{ml})$ & $0.125-2$ & $0.004-0.032$ & $0.38-3$ & $0.016-1.5$ & $0.004-3$ \\
\hline \multirow[t]{3}{*}{ AMC } & MIC50( $\mu \mathrm{g} / \mathrm{mL})$ & 0.5 & 0.016 & 1 & 0.125 & 0.38 \\
\hline & MIC90( $\mu \mathrm{g} / \mathrm{mL})$ & 1 & 0.023 & 1 & 1 & 1 \\
\hline & MIC Range $(\mu \mathrm{g} / \mathrm{ml})$ & $0.094-1.5$ & $0.008-0.023$ & $0.25-2$ & $0.016-1$ & $0.008-2$ \\
\hline \multirow[t]{5}{*}{ CXM } & $1 \%$ & 52.6 & 0 & 66.7 & 36 & 43.8 \\
\hline & $\mathrm{R} \%$ & 36.8 & 0 & 26.7 & 0 & 7.6 \\
\hline & $\operatorname{MIC} 50(\mu \mathrm{g} / \mathrm{mL})$ & 2 & 0.023 & 2 & 0.5 & 1.5 \\
\hline & $\operatorname{MIC90}(\mu \mathrm{g} / \mathrm{mL})$ & 4 & 0.032 & 3 & 2 & 3 \\
\hline & MIC Range $(\mu \mathrm{g} / \mathrm{ml})$ & $0.19-6$ & $0.008-0.125$ & $1-4$ & $0.016-2$ & $0.008-6$ \\
\hline \multirow[t]{5}{*}{ CRO } & $1 \%$ & 15.8 & 0 & 6.7 & 0 & 6.9 \\
\hline & $\mathrm{R} \%$ & 3.5 & 0 & 0 & 0 & 1.4 \\
\hline & $\mathrm{MIC} 50(\mu \mathrm{g} / \mathrm{mL})$ & 0.75 & 0.023 & 0.75 & 0.125 & 0.38 \\
\hline & MIC90( $\mu \mathrm{g} / \mathrm{mL})$ & 1.5 & 0.032 & 1 & 0.5 & 1 \\
\hline & MIC Range( $\mu \mathrm{g} / \mathrm{ml})$ & $0.125-8$ & $0.004-0.047$ & $0.38-1.5$ & $0.016-1$ & $0.004-8$ \\
\hline \multirow[t]{4}{*}{ IPM } & $1 \%$ & 43.9 & 0 & 33.3 & 4 & 49.3 \\
\hline & MIC50( $\mu \mathrm{g} / \mathrm{mL})$ & 0.125 & 0.016 & 0.125 & 0.064 & 0.094 \\
\hline & MIC90( $\mu \mathrm{g} / \mathrm{mL})$ & 0.19 & 0.032 & 0.19 & 0.125 & 0.19 \\
\hline & MIC Range( $\mu \mathrm{g} / \mathrm{ml})$ & $0.047-0.38$ & $0.004-0.064$ & $0.064-0.19$ & $0.012-0.19$ & $0.004-0.38$ \\
\hline \multirow[t]{2}{*}{ TCY } & $1 \%$ & 68.4 & 0 & 6.7 & 4 & 28.5 \\
\hline & $\mathrm{R} \%$ & 5.3 & 95.7 & 93.3 & 80 & 56.9 \\
\hline \multirow[t]{2}{*}{ SXT } & $1 \%$ & 0 & 0 & 0 & 0 & 0 \\
\hline & $\mathrm{R} \%$ & 3.5 & 91.5 & 0 & 92 & 47.2 \\
\hline $\mathrm{CHL}$ & $\mathrm{R} \%$ & 1.8 & 31.9 & 13.3 & 16 & 15.3 \\
\hline
\end{tabular}

PEN penicillin, AMC amoxicillin-clavulanic acid, CXM cefuroxime, CRO ceftriaxone, IPM imipenem, TCY tetracycline, CHL chloramphenicol, SXT trimethoprimsulfamethoxazole, -, no data for disk diffusion test, $I$ intermediate, $R$ resistant, MIC50 minimum inhibitory concentration at which $50 \%$ of the strains were inhibited, MIC90 minimum inhibitory concentration at which $90 \%$ of the strains were inhibited, MIC range range of minimum inhibitory concentration

pressure from antibiotic use which limited the spread of the generally susceptible CC875 at the same time. Previous study suggested that the $\beta$-lactam antibiotics, especially the cephalosporin were frequently used in pediatric clinic work [25]. Chloramphenicol has not been used medically since the 1990s, hence, the resistance rate against Chloramphenicol decreased. Our previous study on serotype $19 \mathrm{~F}$ [9] and $23 \mathrm{~F}$ [10] S. pneumonia isolates indicated that the wide use of beta-lactam antibiotics not only selected the resistant S. pneumonia, but also other epidemiological characteristics, such as serotypes and genotypes. The suggestion is corroborative by the present results.

The genotype replacement of serotype $19 \mathrm{~F}$ and $23 \mathrm{~F} \mathrm{~S}$. pneumonia isolates in China obviously resulted from the input of highly resistant international pneumococcal clones (CC271 and ST81) [9, 10]. Little was known about the spread of serotype $14 S$. pneumonia. There was no sufficient evidence to support that it was associated with some international spread CCs of serotype 14 strains. There were 1594 serotype $14 \mathrm{~S}$. pneumonia isolates in the MLST database (http://spneumoniae.mlst.net/) from the whole world. The eBurst analysis of these data showed that the common CCs were as follows: CC15 (32.3 \%), CC156 (17.8 \% including ST143), CC63 (12.1\%), CC124 (10.7\%) and CC230 (5.7\%). The predominant $\mathrm{CC} 875$ and $\mathrm{CC} 876$ in the present study covered only $30(1.9 \%)$ and $13(0.8 \%)$ strains in the MLST database, respectively. The PMEN notes international spread resistant clones of serotype 14 including ST18, ST9, ST20, ST67, ST230 and ST124, but they were rare in our data.

It was noted that the resistance rate of serotype $14 \mathrm{~S}$. pneumonia isolates to trimethoprim-sulfamethoxazole decreased significantly during the study period. CC876 and CC143 strains were susceptible to trimethoprim- 

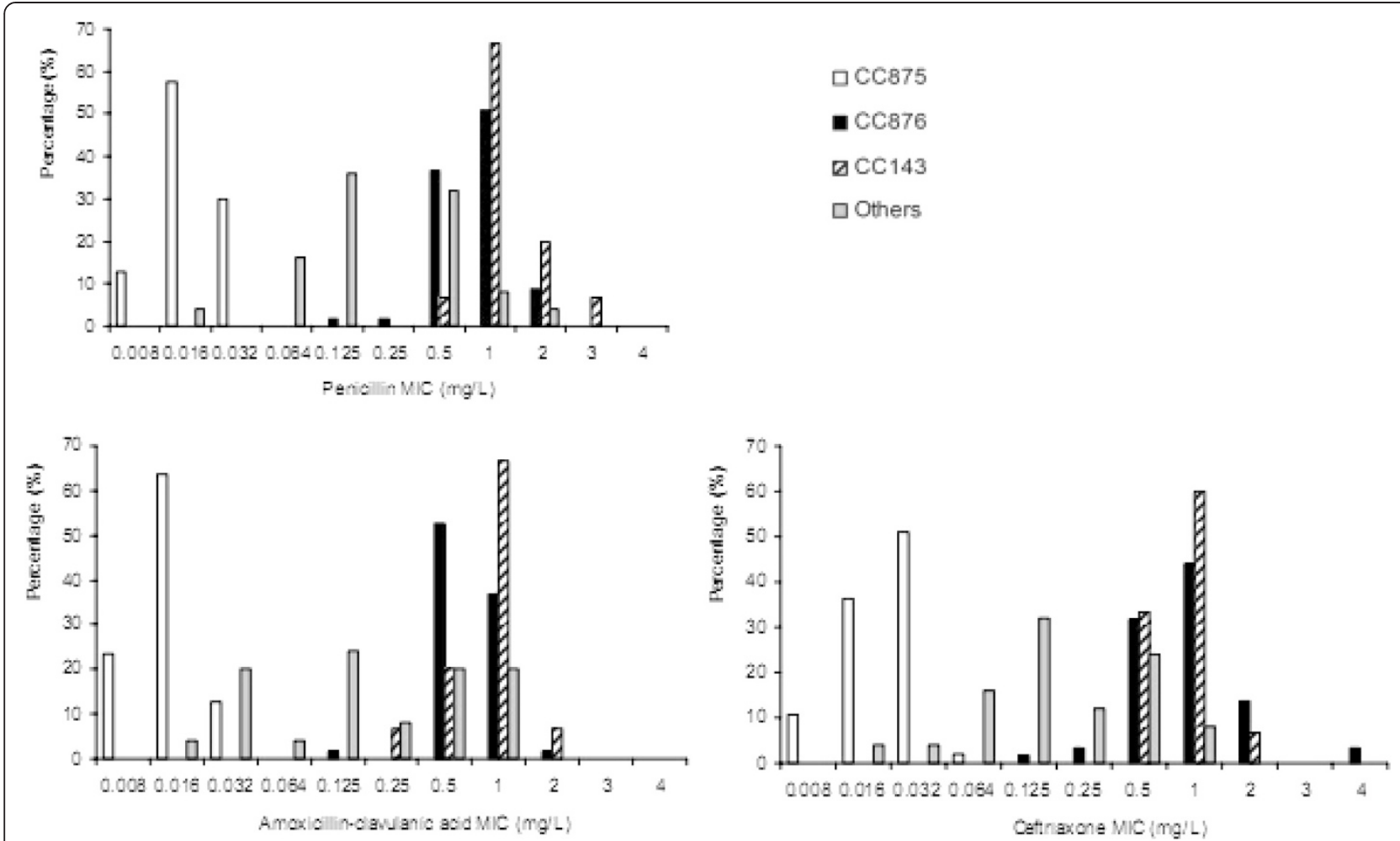

Fig. 3 MIC distribution of the common CCs of serotype 14 S. pneumonia isolates to $\beta$-lactam antibiotics

sulfamethoxazole. We did not find the similar results in serotype $19 \mathrm{~F}$ [9] and $23 \mathrm{~F}$ [10]. The DHFR genes were amplified and sequenced, which confirmed the phenotype test. It was reported [26] that trimethoprim resistance is an essential component of co-trimoxazole resistance. Very few (6\%) of the trimethoprim-resistant isolates were sensitive to co-trimoxazole. A previous study [27] demonstrated that the mutation of Ile100-Leu was critical to the generation of trimethoprim resistance. The results presented here were consistent with the requirement for this mutation but also demonstrated that other changes [28] had considerable impacts on the function of the enzyme. Overall levels of nucleotide divergence from the reference strain were similar in the study, at between 6.0 and $7.5 \%$ for resistant isolates and under $1.5 \%$ for susceptible isolates. The significant epidemiological character should be confirmed by more data.

The study has a limitation. The survey period was divided into five non-equal study period for enough quantity isolates in statistic analysis. Considering the number (144 isolates) of the isolates in this study, we believe that the present result demonstrated the epidemiological changes and molecular characteristics of serotype $14 \mathrm{~S}$. pneumonia in China objectively.

\section{Conclusions}

In summary, we found an increase in the prevalence of serotype $14 \mathrm{~S}$. pneumonia isolates with increasing $\beta$ lactam antibiotic resistance in China from 1997 to 2012. It was indicated that CC876 replaced CC875 under the pressure of antibiotics. Further long-term surveys of serotype $14 \mathrm{~S}$. pneumonia are required to monitor the prevalent STs and antibiotic resistance of this important human pathogen.

\section{Abbreviations}

PCR: Polymerase chain reaction; ST: Sequence type; MLST: Multilocus sequence typing; CC: Clonal complex; MDR: Multidrug resistant; MIC: Minimum inhibitory concentration; DHFR: Dihydrofolate reductase; PCV7: The 7-valent pneumococcal conjugated vaccine; IPD: Invasive pneumococcal disease.

\section{Competing interests}

The authors declare that they have no financial or nonfinancial competing interests related to this manuscript.

\section{Authors' contributions}

$M H, K Y, W S, W G, L Y, S Y$, and YY designed the study. WS and SY performed the antimicrobial susceptibility tests. WG and LY performed the molecular epidemiological PCR. MH, KY, WS and YY collected the data, analyzed them, interpreted the results, and drafted the manuscript. All authors reviewed and revised the manuscript and approved the final version. 


\section{Acknowledgments}

This project was supported by the National Natural Science Foundation of China (Grant No. 81378153), the Capital Health Research and Development of Special (Grant No. 2014-2-1141) and Research Funds of Profession Quota Budjet from Beijing Municipal Science and Technology Commission (2015-bjsekyjs-3).

We sincerely thank the staff of the following esteemed institutions for providing isolates during the several collection programs: Shenzhen Children's Hospital (Yue-jie Zheng, Rui-zhen Zhao), Pediatric Hospital of Fudan University (Li-bo Wang, Chuan-qing Wang), Shengjing Hospital Affiliated to China Medical University (Yun-xiao Shang, Yong Liu), Nanjing Children's Hospital (De-yu Zhao, Hui-yun Wang), Tianjin Children's Hospital (Li-ya Wan), Chongqing Children's Hospital Affiliated to Chongqing Medical University (Lan Liu), Yuying Children's Hospital of Wenzhou Medical College (Changchong Li), Suzhou University Pediatric Hospital (Wei Ji, Yun-fang Ding), Anhui Medical University Affiliated Hospital (Ya-ting Wang, Yuan-hong Xu), First Affiliated Hospital of Xinjiang Medical University (Pei-ru Xu, Ping Ji), Pediatric Hospital of Shanghai Jiao Tong University (Quan Lu, Hong Zhang), Guangzhou Children's Hospital (Li Deng, Qiu-lian Deng), and Xijing Hospital of the Fourth Military Medical University (Guo-cheng Zhang). We are grateful for the use of the MLST database, which is located at Imperial College London and funded by the Welcome Trust.

\section{Received: 31 October 2014 Accepted: 6 July 2015}

\section{Published online: 11 July 2015}

\section{References}

1. World Health Organization. Pneumococcal conjugate vaccine for childhood immunization-WHO position paper. Wkly Epidemiol Rec. 2007:82:93-104.

2. Kattan R, Abu Rayyan A, Zheiman I, Idkeidek S, Baraghithi S, Rishmawi N, et al. Serotype distribution and drug resistance in Streptococcus pneumoniae, Palestinian Territories. Emerg Infect Dis. 2011;17:94-6.

3. Song JH, Jung SI, Ko KS, Kim NY, Son JS, Chang HH, et al. High prevalence of antimicrobial resistance among clinical Streptococcus pneumoniae isolates in Asia (an ANSORP study). Antimicrob Agents Chemother. 2004:48:2101-7.

4. Jauneikaite E, Jefferies JM, Hibberd ML, Clarke SC. Prevalence of Streptococcus pneumoniae serotypes causing invasive and non-invasive disease in South East Asia: a review. Vaccine. 2012;30:3503-14.

5. Dagan $\mathrm{R}$, Klugman KP. Impact of conjugate pneumococcal vaccines on antibiotic resistance. Lancet Infect Dis. 2008;8:785-95.

6. Feikin DR, Klugman KP. Historical changes in pneumococcal serogroup distribution: implications for the era of pneumococcal conjugate vaccines. Clin Infect Dis. 2002;35:547-55.

7. García-Suárez Mdel M, Villaverde R, Caldevilla AF, Méndez FJ, Vázquez F. Serotype distribution and antimicrobial resistance of invasive and noninvasive pneumococccal isolates in Asturias, Spain. Jpn J Infect Dis. 2006;59:299-305.

8. Liu C, Xiong X, Xu W, Sun J, Wang L, Li J. Serotypes and patterns of antibiotic resistance in strains causing invasive pneumococcal disease in children less than 5 years of age. PLoS One. 2013;8:e54254.

9. Li QH, Yao KH, Yu SJ, Ma X, He MM, Shi W, et al. Spread of multidrugresistant clonal complex 271 of serotype $19 \mathrm{~F}$ Streptococcus pneumoniae in Beijing, China: characterization of serotype 19 F. Epidemiol Infect. 2013;141:2492-6.

10. Ma X, Yao KH, Yu SJ, Zhou L, Li QH, Shi W, et al. Genotype replacement within serotype $23 \mathrm{~F}$ Streptococcus pneumoniae in Beijing, China: characterization of serotype 23 F. Epidemiol Infect. 2013;141:1690-6.

11. Li J, Yang YH, Yu SJ, Yuan L, Hu YY. Study on resistance to antibiotics to Streptococcus pneumoniae. Zhonghua Erke Zazhi. 2011;37:408-11 (in Chinese).

12. Xue L, Yao K, Xie G, Zheng Y, Wang C, Shang Y, et al. Serotype distribution and antimicrobial resistance of Streptococcus pneumoniae isolates that cause invasive disease among Chinese children. Clin Infect Dis. 2010;50:741-4.

13. Yao K, Shen X, Yu S, Lu Q, Deng L, Ye Q, et al. Antimicrobial resistance and serotypes of nasopharyngeal strains of Streptococcus pneumoniae in Chinese children with acute respiratory infections. J Int Med Res. 2007;35:253-67.

14. Yao KH, Wang LB, Zhao GM, Zheng YJ, Deng L. Study on antimicrobial resistance in different serotypes of Streptococcus pneumoniae isolated from pediatric patients hospitalized for pneumonia. Linchuang Erke Zazhi. 2009;27:524-7. 533 (in Chinese).

15. Yu J, Carvalho Mda G, Beall B, Nahm MH. A rapid pneumococcal serotyping system based on monoclonal antibodies and PCR. J Med Microbiol. 2008:57:171-8.

16. Ma X, Zhao R, Ma Z, Yao K, Yu S, Zheng Y, et al. Serotype distribution and antimicrobial resistance of Streptococcus pneumoniae isolates causing invasive diseases from Shenzhen children's hospital. PLoS One. 2013;8:e67507

17. Kelly LM, Jacobs MR, Appelbaum PC. Comparison of agar dilution, microdilution, E-test, and disk diffusion methods for testing activity of cefditoren against Streptococcus pneumoniae. J Clin Microbiol. 1999;37:3296-9.

18. Clinical Laboratory Standards Institute (CLSI). Performance standards for antimicrobial susceptibility testing; twenty-second informational supplement, M100-S22. Wayne, PA: CLSI; 2012.

19. Sutcliffe J, Grebe T, Tait-Kamradt A, Wondrack L. Detection of erythromycin-resistant determinants by PCR. Antimicrob Agents Chemother. 1996;40:2562-6.

20. Enright MC, Spratt BG. A multilocus sequence typing scheme for Streptococcus pneumoniae: identification of clones associated with serious invasive disease. Microbiology. 1998;144:3049-60.

21. Maskell JP, Sefton AM, Hall LM. Multiple mutations modulate the function of dihydrofolate reductase in trimethoprim-resistant Streptococcus pneumoniae. Antimicrob Agents Chemother. 2001:45:1104-8.

22. Yao KH, Yang YH. Streptococcus pneumoniae diseases in Chinese children: past, present and future. Vaccine. 2008;26:4425-33.

23. Liu Y, Wang $H$, Chen $M$, Sun Z, Zhao R, Zhang L, et al. Serotype distribution and antimicrobial resistance patterns of Streptococcus pneumoniae isolated from children in China younger than 5 years. Diagn Microbiol Infect Dis. 2008;61:256-63.

24. Antonio M, Dada-Adegbola H, Biney E, Awine T, O'Callaghan J, Pfluger V, et al. Molecular epidemiology of pneumococci obtained from Gambian children aged 2-29 months with invasive pneumococcal disease during a trial of a 9-valent pneumococcal conjugate vaccine. BMC Infect Dis. 2008;8:81.

25. Denham BC, Clarke SC. Serotype incidence and antibiotic susceptibility of Streptococcus pneumoniae causing invasive disease in Scotland, 1999-2002. J Med Microbiol. 2005;54:327-31.

26. Zhang W, Shen X, Wang Y, Chen Y, Huang M, Zeng Q, et al. Antibiotic use in five children's hospitals during 2002-2006: the impact of antibiotic guidelines issued by the Chinese Ministry of Health. Pharmacoepidemiol Drug Saf. 2008;17:306-11.

27. Adrian PV, Klugman KP. Mutations in the dihydrofolate reductase gene of trimethoprim-resistant isolates of Streptococcus pneumoniae. Antimicrob Agents Chemother. 1997;41:2406-13.

28. Pikis A, Donkersloot JA, Rodriguez WJ, Keith JM. A conservative amino acid mutation in the chromosome-encoded dihydrofolate reductase confers trimethoprim resistance in Streptococcus pneumoniae. J Infect Dis. 1998;178:700-6.

\section{Submit your next manuscript to BioMed Central and take full advantage of:}

- Convenient online submission

- Thorough peer review

- No space constraints or color figure charges

- Immediate publication on acceptance

- Inclusion in PubMed, CAS, Scopus and Google Scholar

- Research which is freely available for redistribution 